\title{
Undecidability of Chop
}

\author{
Angelo Montanari *, Emilio Muñoz-Velasco ${ }^{\dagger}$, Guido Sciavicco ${ }^{\ddagger}$, \\ * Department of Mathematics and Computer Science \\ University of Udine, Italy - Email: angelo.montanari@uniud.it \\ $\dagger$ Department of Applied Mathematics \\ University of Malaga, Spain - Email: emilio@ctima.uma.es \\ $\ddagger$ Department of Information Engineering and Communications \\ University of Murcia, Spain - Email: guido@um.es
}

\begin{abstract}
The chop operator $C$ is a binary modality that plays an important role in interval temporal logics. Such an operator, which is not definable in Halpern and Shoham's modal logic of time intervals $\mathrm{HS}$, allows one to split an interval into two parts and to specify what is true over them. $C$ appears both in Moszkowski's PITL (that pairs it with a modal constant $\pi$ which is true on all and only the intervals with coincident endpoints) and in Venema's CDT (that also features the binary modalities $D$ and $T$, and $\pi$ ). Without the so-called locality principle, which restricts the semantics of proposition letters, the satisfiability problem for both PITL and CDT turns out to be undecidable over all meaningful classes of linear orders. The problem has been shown to be undecidable also for the fragment $C$, that is, PITL without $\pi$, over infinite linear orders. In this paper, we prove that the same holds for $C$ over finite linear orders. To this end, we exploit the close relation between $C$ and the reflexive version of the $H S$ fragment $B E_{H S}$, whose modalities correspond to Allen's relations starts and finishes: we prove that the satisfiability problem for reflexive $B E_{H S}$ is undecidable; undecidability of the same problem for $C$ comes as a corollary.
\end{abstract}

Keywords-Interval Temporal Logics; Satisfiability Checking; Decidability.

\section{INTRODUCTION}

Time intervals, rather than time points, are regarded as the primitive ontological entities in interval temporal logics (ITLs for short). The truth of ITL formulae is defined accordingly. ITLs turn out to be useful in many fields, including hardware [20] and real-time system [27] verification, language processing [23], and constraint satisfaction and planning [1]. Moreover, ITLs have been considered as the basis for temporal extensions of Description Logics [4], as witnessed by various contributions over the years, e.g. [2], [3], [5], [24].

Among the most influential ITLs there are Mozskowski's PITL [20], Halpern and Shoham's modal logic of Allen's Relations HS [11], and Venema's CDT [26]. CDT subsumes both HS and PITL, and it inherits their bad computational behaviour. As a matter of fact, HS is highly undecidable over all meaningful classes of linear orders; a few decidable syntactic fragments have been identified in the last years, whose complexities range from NP-complete to
NEXPTIME-complete, EXPSPACE-complete, and even nonprimitive recursive (see, among others, [6], [7], [8], [9], [17], [18], [19]).

The semantics of the chop modality $C$ can be described as follows: given a pair of formulae $\phi$ and $\psi$, and an interval $[x, y], \phi C \psi$ holds over $[x, y]$ if and only if we can split it into a $\phi$-part followed by a $\psi$-part (see Fig. 1). ITLs with chop, such as, for instance, CDT, are of both theoretical and practical interest. On the theoretical side, Venema showed that, over linearly ordered sets, CDT is expressively complete for the fragment of first-order logic with 3 variables (at most 2 of which are free), with equality, the pre-interpreted binary relation $<$, and uninterpreted binary relations [26]. Moreover, he pointed out and explored the close link between CDT and Relation Algebras [13]. On the application side, the chop operator has proved itself very useful in various contexts, including verification of access control policies in PITL [10], compositional reasoning in PITL [21], [22], and program verification in CDT [26].

Undecidability of (the satisfiability problem for) CDT motivated the analysis of the computational behaviour of its one-modality fragments C, D, and T. In [12], Hodkison et al. showed that $\pi$ (a modal constant which evaluates to true precisely on intervals with coincident endpoints) is not expressible by the modalities $C, D$, and $T$. Moreover, they proved that the fragments $D$ and $T$ turn out to be undecidable over all classes of linear orders, with the exception of the class of all finite orders and $\mathbb{N}$ (resp. $\mathbb{Z}^{-}$) for which the problem was left open. As for the fragment C, they only showed that it is undecidable over the classes of all linear orders and of all dense linear orders. Undecidability of $\mathrm{C}+\pi$ over the class of finite linear orders, the class of (strongly) discrete linear orders, $\mathbb{N}$, and $\mathbb{Z}$ (as a matter of fact, $\mathrm{C}+\pi$ is not able to distinguish among these classes, as it only allows one to look inside the initial interval) was known from [20]. The problem of establishing whether C (without $\pi$ ) is decidable or not in the finite/discrete case was left open.

As noticed by Lodaya in [14], the fragment $C$ is closely related to the $\mathrm{HS}$ fragments $\mathrm{BE}_{\mathrm{HS}}$ and $\mathrm{D}_{\mathrm{HS}}$. The former features two modalities $\langle B\rangle$ and $\langle E\rangle$ for Allen's relations 


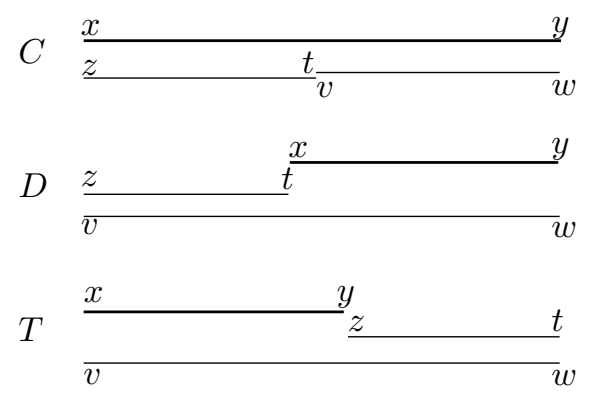

Figure 1. A pictorial account of the semantics of $C, D$, and $T$ (the thick line represents the current interval $[x, y]$ ).

starts and finishes, respectively, while the latter has only one modality for the relation during. $\mathrm{BE}_{\mathrm{HS}}$ is definable in $\mathrm{C}+\pi$, while $C$ (devoid of $\pi$ ) is able to express both $\langle B\rangle$ and $\langle E\rangle$ in their reflexive version. Reflexive $\mathrm{BE}_{\mathrm{HS}}$, in turn, allows one to define the reflexive version of $\langle D\rangle$. The standard, irreflexive version of $D_{\mathrm{HS}}$ is undecidable in the finite/discrete case [15], implying the undecidability of irreflexive $B_{H S}$. On the contrary, the reflexive version of $D_{H S}$ turns out to be PSPACE-complete when interpreted over finite/discrete linear orders [16], leaving the status of the satisfiability problem for reflexive $B_{\mathrm{HS}}$ as an open problem.

In this paper, we solve this latter problem: we prove the undecidability of reflexive $\mathrm{BE}_{\mathrm{HS}}$ in the finite/discrete case by a reduction from the halting problem for Turing Machines with an empty input. The undecidability of $C$ in the finite/discrete case comes as a corollary. In addition, we show how to generalize the proposed reduction to the class of all linear orders and the class of all dense linear orders. The (already known) undecidability of $C$ in these cases [12] comes, again, as a corollary.

\section{PRELIMINARIES}

The language of Venema's CDT [26] consists of a denumerable set of proposition letters $\mathbf{P}$, the Boolean connectives $\wedge$ and $\neg$ (the other ones can be viewed as abbreviations), the modal constant $\pi$, and the three binary modalities $C, D$, and $T$ ( $C$ stands for 'chop'; one may think of $D$ and $T$ as shorthands for 'Done' and 'To come', respectively).

Well-formed CDT formulae, denoted by $\varphi, \psi, \ldots$, are generated by the following grammar:

$\varphi:=p|\neg \varphi| \varphi \wedge \psi|\pi| \varphi C \psi|\varphi D \psi| \varphi T \psi$, with $p \in \mathbf{P}$

For each $\mathrm{S} \subseteq\{C, D, T\}$, we denote by $\mathrm{S}$ (resp., $\mathrm{S}+\pi$ ) the fragment of CDT that only features modalities in $S$ and does not include (resp., includes) the modal constant $\pi$. According to such a notation, hereafter we will write CDT $+\pi$ for CDT, and we will denote by CDT the fragment devoid of $\pi$.
Let $\mathbb{D}=\langle D,<\rangle$ be an (irreflexive) linearly ordered set. A non-strict (resp., strict) interval over $\mathbb{D}$ is an ordered pair $[x, y]$, where $x, y \in D$ and $x \leq y$ (resp., $x<y$ ). Accordingly, non-strict (resp., strict) semantics evaluates formulae over non-strict (resp., strict) intervals. Allen's Interval Algebra [1] assumes strict semantics, Halpern and Shoham's HS [11] non-strict one. Venema's CDT $+\pi$ was proposed as a generalization of $\mathrm{HS}$, and thus it assumes non-strict semantics as well. The semantics of CDT $+\pi$ is given in terms of interval models $M=\langle\mathbb{I}(\mathbb{D}), V\rangle$, where $\mathbb{I}(\mathbb{D})$ is the set of all non-strict intervals over $\mathbb{D}$ and $V$ is a valuation function $V: \mathbf{P} \mapsto 2^{\mathbb{I}(\mathbb{D})}$, that assigns to each atomic proposition $p \in \mathbf{P}$ the set of intervals $V(p)$ on which $p$ holds. Truth of formulae on an interval $[x, y]$ in a model $M$ is defined by structural induction on formulae. While proposition letters and Boolean operators are defined in the standard way, the semantic rules for the modal operators are as follows:

- $M,[x, y] \Vdash \pi$ if and only if $x=y$;

- $M,[x, y] \Vdash \varphi C \psi$ if and only if there exist two intervals $[z, t],[v, w]$ such that $x=z, t=v, y=w, M,[z, t] \Vdash$ $\varphi$, and $M,[v, w] \Vdash \psi$;

- $M,[x, y] \Vdash \varphi D \psi$ if and only if there exist two intervals $[z, t],[v, w]$ such that $z=v, x=t, y=w, M,[z, t] \Vdash$ $\psi$, and $M,[v, w] \Vdash \varphi$;

- $M,[x, y] \Vdash \varphi T \psi$ if and only if there exist two intervals $[z, t],[v, w]$ such that $x=v, y=z, t=w, M,[z, t] \Vdash$ $\psi$, and $M,[v, w] \Vdash \varphi$.

A graphical account of the semantics of the three modalities $C, D$, and $T$ is given in Fig. 1 .

Halpern and Shoham's HS features a (unary) modal operator for each (binary) Allen relation. For $X \in$ $\{A, L, B, E, O, D\}$, its formulae are generated by the following grammar:

$$
\varphi:=p|\neg \varphi| \varphi \wedge \psi|\langle X\rangle \psi|\langle\bar{X}\rangle \psi \text {, with } p \in \mathbf{P}
$$

In particular, modalities $\langle B\rangle$ and $\langle E\rangle$ correspond to Allen's relations starts and finishes, respectively, and, along with the transposed modalities $\langle\bar{B}\rangle$ and $\langle\bar{E}\rangle$, are semantically defined as follows:

- $M,[x, y] \Vdash\langle B\rangle \psi$ if and only if there exists an interval $[z, t]$ such that $x=z, x \leq t<y$, and $M,[z, t] \Vdash \psi$;

- $M,[x, y] \Vdash\langle\bar{B}\rangle \psi$ if and only if there exists an interval $[z, t]$ such that $x=z, t>y$, and $M,[z, t] \Vdash \psi$;

- $M,[x, y] \Vdash\langle E\rangle \psi$ if and only if there exists an interval $[z, t]$ such that $t=y, x<z \leq y$, and $M,[z, t] \Vdash \psi$;

- $M,[x, y] \Vdash\langle\bar{E}\rangle \psi$ if and only if there exists an interval $[z, t]$ such that $t=y, z<x$, and $M,[z, t] \Vdash \psi$.

The fragment of HS featuring $\langle B\rangle$ and $\langle E\rangle$ is denoted by $\mathrm{BE}_{\mathrm{HS}}$, while the fragment of $\mathrm{HS}$ with the modality $\langle D\rangle$ only is denoted by $\mathrm{D}_{\mathrm{HS}}$. 
It can be easily checked that $\langle D\rangle \psi$ can be defined as $\langle B\rangle\langle E\rangle \psi$ in $\mathrm{BE}_{\mathrm{HS}}$ and that $\langle B\rangle \psi$ and $\langle E\rangle \psi$ can be respectively defined as $\psi C \neg \pi$ and $\neg \pi C \psi$ in $\mathrm{C}+\pi$. Moreover, it is possible to prove that $\langle B\rangle$ and $\langle E\rangle$ are not definable in $\mathrm{D}_{\mathrm{HS}}$ and $C$ is not definable $\mathrm{BE}_{\mathrm{HS}}$. It immediately follows that $\mathrm{D}_{\mathrm{HS}} \prec \mathrm{BE}_{\mathrm{HS}} \prec \mathrm{C}+\pi$, where $\mathrm{L} \prec \mathrm{L}^{\prime}$ reads as $\mathrm{L}^{\prime}$ embeds $\mathrm{L}$, but not vice versa.

The reflexive versions of $\mathrm{BE}_{\mathrm{HS}}$ and $\mathrm{D}_{\mathrm{HS}}$ are obtained from the original ones by replacing strict linear order $<$ by the non-strict one $\leq$. It still holds that $D_{\mathrm{HS}} \prec \mathrm{BE}_{\mathrm{HS}}$; moreover, it holds that (reflexive) $\mathrm{BE}_{\mathrm{HS}} \prec \mathrm{C}$. Notice that irreflexive $\langle D\rangle$ is definable in $\mathrm{C}+\pi$, but not in $\mathrm{C}$. The same for irreflexive $\langle B\rangle$ and $\langle E\rangle$.

In the following, we focus our attention on reflexive $B E_{H S}$, and we prove that its satisfiability problem over finite/discrete linear orders is undecidable ${ }^{1}$, in sharp contrast with the PSPACE-completeness of the satisfiability problem for reflexive $D_{H S}$ over finite/discrete linear orders [16]. The undecidability of C, over finite/discrete linear orders, comes as an immediate corollary.

\section{UNDECIDABILITY OF C: THE FINITE/DISCRETE CASE}

In this section, we prove the main result of the paper: the undecidability of $C$ in the finite/discrete case. As we already pointed out, $\pi$ is not definable in CDT, and thus in C. This is not the case with (some fragments of) standard irreflexive HS. As an example, $\pi$ can be defined in the HS fragment $\mathrm{B}$ by the constant formula $[B] \perp$, where $[B]$ is a shorthand for $\neg\langle B\rangle \neg \perp$, which evaluates to true on point-intervals only. Now, one of the distinctive feature of $C$, when interpreted in a linear order, is that its semantics is inherently reflexive: one way of satisfying the formula $\phi C \psi$ over an interval $[x, y]$ is having $\phi$ true over $[x, y]$ and $\psi$ true over the point-interval $[y, y]$. The lack of $\pi$ makes it impossible to exclude such an intepretation (in $\mathrm{C}+\pi$, we can exclude it with the formula $\phi C(\neg \pi \wedge \psi))$. This makes it difficult to force the existence of a chain of consecutive intervals with a constant formula, which is a common ingredient of undecidability proofs in the field of interval temporal logic. In [12], Hodkinson et al. provide a formula of $\mathrm{C}$ that forces the existence of such a chain, but it only works (that is, is satisfiable) when the chain is infinite. In this paper, we show that a possibly (but not necessarily) infinite chain can be forced in reflexive $B E_{H S}$ by applying a more sophisticated technique.

To start with, we recall the basic characteristics of Turing machines. A Turing Machine is a tuple $\mathcal{M}_{T}=$ $\left(Q, \Sigma, \Gamma, \delta, q_{0}, q_{f}\right)$, where $Q$ is a finite set of states, $q_{0}$ (resp., $q_{f}$ ) is the initial (resp., final) state, $\Sigma$ is the machine alphabet, that does not contain the symbol $\sqcup$ (blank), $\Gamma=\Sigma \cup\{\sqcup\}$ is the tape alphabet, and $\delta: Q \times \Gamma \rightarrow Q \times \Gamma \times\{L, R\}$

\footnotetext{
${ }^{1}$ By discrete linear orders we mean those linear orders where there exists a finite number of points, possibly no one, between any two distinct points (often called strongly discrete linear orders in the literature).
}

is the transition function ( $L$, for left, and $R$, for right, are the possible moves on the machine tape). It is well known that both the halting and the non-halting problems for a deterministic Turing Machine are undecidable (the former is R.E.-complete, while the latter is CO-R.E.-complete). In the following, we first provide a reduction of the halting problem for Turing machines to the satisfiability problem for reflexive $B E_{H S}$, when the underlying linear order is discrete, and then we show how to revise it in order to obtain a reduction from the non-halting problem, with no discreteness assumption. Observe that for the finite/discrete case, as well as for infinite Dedekind-complete linear orders, our results imply that both reflexive $B_{H S}$ and $C$ are not recursively axiomatizable.

The key step of the construction is the representation of the computation history of a Turing Machine $\mathcal{M}_{T}$. We interpret every interval $[x, y]$ as a point $(x, y)$ of the Cartesian half-plane $x \leq y$ (the so-called Compass Structure [25]), and we provide interval modalities with a spatial semantics. The spatial counterparts of Allen's relations begins and ends are the relations down and right on the Cartesian plane, respectively. We identify a strictly increasing finite sequence $x_{0}, x_{1}, \ldots, x_{m}$ and a strictly decreasing finite sequence $y_{0}, y_{1}, \ldots, y_{m}$, that define a finite grid, and then we exploit such a grid to encode the computation history of $\mathcal{M}_{T}$. Let

$$
U \varphi \equiv[B][E] \varphi
$$

be a (definable) universal modality that forces $\varphi$ to be true everywhere in the current interval. We build the grid by constraining every interval of the model to be labeled with a proposition letter $h$ (resp., $v$ ), or its negation, as follows:

$$
\begin{aligned}
\phi_{1}= & h \wedge v \wedge U(h \rightarrow[B] h) \wedge U(\neg h \rightarrow[B] \neg h) \\
& \wedge U(v \rightarrow[E] v) \wedge U(\neg v \rightarrow[E] \neg v) .
\end{aligned}
$$

Notice that $\phi_{1}$ labels the endpoints of an interval with $h$ and $v$, respectively, and thus a form of locality principle has been considered for these proposition letters. The role of $h$ and $v$ can be formally expressed by the notions of horizontal set $\operatorname{set}_{h}(x)$ and vertical set $\operatorname{set}_{v}(y)$, which are defined as follows.

Definition 1: Let $M$ be a model and $[x, y]$ be an interval such that $M,[x, y] \Vdash \phi_{1}$. For any $z \in D$, with $x \leq z \leq y$, we define $\operatorname{set}_{h}(z)$ as the maximal subset of $D$ such that: (i) $z \in \operatorname{set}_{h}(z)$; (ii) if $s, t \in \operatorname{set}_{h}(z)$, then $u \in \operatorname{set}_{h}(z)$, for all $s \leq u \leq t$; (iii) for all $s, s^{\prime} \in \operatorname{set}_{h}(z)$, and $t \in D$, with $x \leq t \leq y, M,[s, t] \Vdash h$ if and only if $M,\left[s^{\prime}, t\right] \Vdash h$. The notion of $\operatorname{set}_{v}(z)$ is defined in a symmetric way.

With a little abuse of notation, for any $s \in D$ and $\sim \in\{<$, $\leq,>, \geq\}$, we write $s \sim \operatorname{set}_{h}(z)$ if (and only if) $s \sim t$ for all $t \in \operatorname{set}_{h}(z)$. Obviously, $s \sim \operatorname{set}_{h}(z)$ implies $s \sim z$, but not the other way around. Now, we show how to express the immediate right- and down-successor of a point $\left(x_{i}, y_{j}\right)$ in the grid by means of the shortcuts $I_{r i}(p, q)$ and $I_{d o}(p, q)$ 
below, where $p$ and $q$ are proposition letters. $I_{r i}(p, q)$ is the conjunction of the universal formulae $\tau_{1}, \ldots, \tau_{6}$ and the formulae obtained from them by replacing each occurrence of $h$ (resp., $\neg h$ ) by $\neg h$ (resp., $h$ ):

$$
\begin{aligned}
& \tau_{1}=U((p \wedge h) \rightarrow\langle E\rangle(q \wedge \neg h)) \\
& \tau_{2}=U\left((p \wedge h) \rightarrow[E] p^{\prime}\right) \wedge U\left(\left(p^{\prime} \wedge \neg h\right) \rightarrow[E] \neg p\right) \\
& \tau_{3}=U\left((q \wedge \neg h) \rightarrow[E] q^{\prime}\right) \wedge U\left(\left(q^{\prime} \wedge h\right) \rightarrow[E] \neg q\right), \\
& \tau_{4}=U\left(p^{\prime} \wedge h \wedge\langle E\rangle(q \wedge \neg h) \rightarrow p\right) \\
& \tau_{5}=U\left(\left(p^{\prime} \wedge \neg h \wedge\langle E\rangle q\right) \rightarrow q\right) \\
& \tau_{6}=U((q \wedge\langle E\rangle p) \rightarrow \perp),
\end{aligned}
$$

where, for every occurrence of $I_{r i}(p, q), p^{\prime}$ and $q^{\prime}$ are auxiliary fresh proposition letters.

Lemma 1: Let us assume that $M,[x, y] \Vdash \phi_{1}$. Then, for all $z, t$ such that $x \leq z \leq t \leq y$ and $M,[z, t] \Vdash I_{r i}(p, q) \wedge p$, there exists $s>\operatorname{set}_{h}(z)$ such that $M,[s, t] \Vdash q$. Furthermore, if $M,[z, t] \Vdash h$ (resp., $M,[z, t] \Vdash \neg h$ ), then $M,[s, t] \Vdash \neg h$ (resp., $M,[s, t] \Vdash h$ ), and:

1) for each $s^{\prime}>\operatorname{set}_{h}(z), M,\left[s^{\prime}, t\right] \Vdash \neg p$;

2) for each $s^{\prime}>\operatorname{set}_{h}(s), M,\left[s^{\prime}, t\right] \Vdash \neg q$;

3) for each $s^{\prime} \in \operatorname{set}_{h}(z), s^{\prime} \geq z, M,\left[s^{\prime}, t\right] \Vdash p$;

4) for each $s^{\prime} \in \operatorname{set}_{h}(s), s^{\prime} \leq s, M,\left[s^{\prime}, t\right] \Vdash q$;

5) for each $z \leq s^{\prime} \leq s$, either $s^{\prime} \in \operatorname{set}_{h}(z)$ or $s^{\prime} \in \operatorname{set}_{h}(s)$.

Proof: Assume $M,[z, t] \Vdash p \wedge h$ (the case in which $M,[z, t] \Vdash p \wedge \neg h$ can be dealt with in a similar way). By formula $\tau_{1}$, there exists $s>z$ such that $M,[s, t] \Vdash q \wedge \neg h$. By definition of $\operatorname{set}_{h}(z), s>\operatorname{set}_{h}(z)$. Now, if $s^{\prime}>\operatorname{set}_{h}(z)$, then there exists $z<s^{\prime \prime} \leq s$ such that $M,\left[s^{\prime \prime}, t\right] \Vdash \neg h$. By formula $\tau_{2}$, it holds that $M,\left[s^{\prime \prime}, t\right] \Vdash p^{\prime}$ and, for each $s^{\prime \prime} \leq s^{\prime \prime \prime} \leq t, M,\left[s^{\prime \prime \prime}, t\right] \Vdash \neg p$; therefore, $M,\left[s^{\prime}, t\right] \Vdash \neg p$, thus proving (1). Consider now a point $s^{\prime}>\operatorname{set}_{h}(s)$. Since $M,[s, t] \Vdash q \wedge \neg h$, there exists $s<s^{\prime \prime} \leq s^{\prime}$ such that $M,\left[s^{\prime \prime}, t\right] \Vdash h$. By the very same argument used to prove (1), we can show that formula $\tau_{3}$ implies $M,\left[s^{\prime}, t\right] \Vdash \neg q$, thus proving (2). Let $s^{\prime} \in \operatorname{set}_{h}(z)$ such that $s^{\prime} \geq z$. Then, $M,\left[s^{\prime}, t\right] \Vdash h$ and, by formula $\tau_{2}, M,\left[s^{\prime}, t\right] \Vdash p^{\prime}$. Since $s^{\prime}<s$, we have that $M,\left[s^{\prime}, t\right] \Vdash\langle E\rangle(q \wedge \neg h)$, and then, by formula $\tau_{4}, M,\left[s^{\prime}, t\right] \Vdash p$, thus proving (3). Consider now some $s^{\prime} \in \operatorname{set}_{h}(s)$ such that $s^{\prime} \leq s$. Since $M,[s, t] \Vdash$ $q \wedge \neg h$, it holds that $M,\left[s^{\prime}, t\right] \Vdash \neg h \wedge\langle E\rangle q$. Moreover, since $M,[z, t] \Vdash p \wedge h$ and $s^{\prime}>z$, by formula $\tau_{2}$, we have that $M,\left[s^{\prime}, t\right] \Vdash p^{\prime}$. Hence, we can apply formula $\tau_{5}$ to deduce that $M,\left[s^{\prime}, t\right] \Vdash q$, thus proving (4). Finally, let $s^{\prime}$ be such that $z \leq s^{\prime} \leq s$. If $M,\left[s^{\prime}, t\right] \Vdash h$, then, by formula $\tau_{2}$, $M,\left[s^{\prime}, t\right] \Vdash p^{\prime} \wedge\langle E\rangle(q \wedge \neg h)$. Therefore, by formula $\tau_{4}$, $M,\left[s^{\prime}, t\right] \Vdash p$ and, by item (1), we can conclude that $s^{\prime} \in$ $\operatorname{set}_{h}(z)$. If $M,\left[s^{\prime}, t\right] \Vdash \neg h$, assume, by way of contradiction, that $s^{\prime} \notin \operatorname{set}_{h}(s)$. Then, there exists $s^{\prime \prime}$, with $s^{\prime}<s^{\prime \prime}<$ $s$, such that $M,\left[s^{\prime \prime}, t\right] \Vdash h$. Now, since $M,\left[s^{\prime}, t\right] \Vdash \neg h$, by formula $\tau_{2}$, it holds that $M,\left[s^{\prime}, t\right] \Vdash p^{\prime} \wedge\langle E\rangle q$ and, by formula $\tau_{5}$, it holds that $M,\left[s^{\prime}, t\right] \Vdash q \wedge \neg h$. By formula $\tau_{3}$, it follows that $M,\left[s^{\prime \prime}, t\right] \Vdash q^{\prime}$ and $M,[s, t] \Vdash \neg q$, which is a contradiction, thus proving (5).
The shortcut $I_{d o}(p, q)$ is obtained from $I_{r i}(p, q)$ by replacing each occurrence of $h$ by $v, \neg h$ by $\neg v,\langle E\rangle \varphi$ by $\langle B\rangle \varphi$, and $[E] \varphi$ by $[B] \varphi$, and the above lemma can be restated accordingly.

On the basis on Lemma 1 and the corresponding lemma for $I_{d o}(p, q)$, and taking into account that we are in the discrete/finite setting, we can give the following definition.

Definition 2: Let $M,[x, y] \Vdash \phi_{1}$. Then, for every $x \leq$ $z \leq y$, let (i) $\operatorname{next}_{h}(z)=\min \left\{s \mid s>\operatorname{set}_{h}(z)\right\}$ and (ii) $\operatorname{next}_{v}(z)=\max \left\{s \mid s<\operatorname{set}_{v}(z)\right\}$.

Notice that, by Lemma 1 , if $M,[x, y] \Vdash \phi_{1}$ and $M,[z, t] \Vdash$ $I_{r i}(p, q) \wedge p$, then $M,\left[\operatorname{next}_{h}(z), t\right] \Vdash q$. Similarly, if $M,[z, t] \Vdash I_{d o}(p, q) \wedge p$, then $M,\left[z, \operatorname{next}_{v}(t)\right] \Vdash q$.

We are now ready to start the encoding of the computation history of a Turing machine. The plan of the construction can be summarized as follows: (i) tape and auxiliary symbols are placed on $u$-intervals (intervals labeled by the proposition letter $u$, later referred to as units); (ii) the symbol $*$ labels those $u$-intervals that represent cells of the tape (later called cells); (iii) the proposition letters $D o, U p$, and $A b$ encode the correspondences between symbols on the tape from one configuration to the next one, while $\mathrm{Ne}$ deals with neighboring symbols on the same configuration; (iv) the proposition letter Stop is used to place the last configuration which contains the final state $q_{f}$. We use also $N e$ ?, $U p$ ? and $A b$ ? in order to ensure that the sequence of $u$-intervals has to continue until the last configuration has been constructed. The $u$-intervals will be placed on a segment starting with the initial interval $[x, y]$ and ending on the diagonal of the first quadrant at some $z$ such that $x \leq z \leq y$ (see Fig. 2). To represent the situation of $\mathcal{M}_{T}$ at a given moment of the computation, we define $\mathcal{M}_{T}$-configurations, that include the content of the tape, the position of the reading head, and the current state. Moreover, we distinguish between $\mathcal{M}_{T}$-configurations and, simply, configurations: the latter indicate the portion of the model that represent the former in the construction. Those units that help us to separate one configuration from the next one will be denoted by $*$, and we will use 0,1 , and $\sqcup$ to represent tape cells not under the machine's head, and the propositional symbols $q^{c}$, with $q \in Q \backslash\left\{q_{f}\right\}$ and $c \in\{0,1, \sqcup\}$, to represent the tape cell under the head and the current (non-final) state of the machine. Let us define:

$$
\mathbf{L}=\{0,1, \sqcup, *\} \cup\left\{q^{c} \mid q \in Q \wedge c \in\{0,1, \sqcup\}\right\} \cup\left\{q_{f}\right\} .
$$

By a little abuse of notation, we assume that all symbols $q_{f}^{c}$ are equal to $q_{f}$. To prove our result, we reduce the halting problem for a deterministic Turing machine $\mathcal{M}_{T}$ with a tape infinite to the left and finite to the right. The chain of $u$ intervals, the first configuration, and the position of the final 


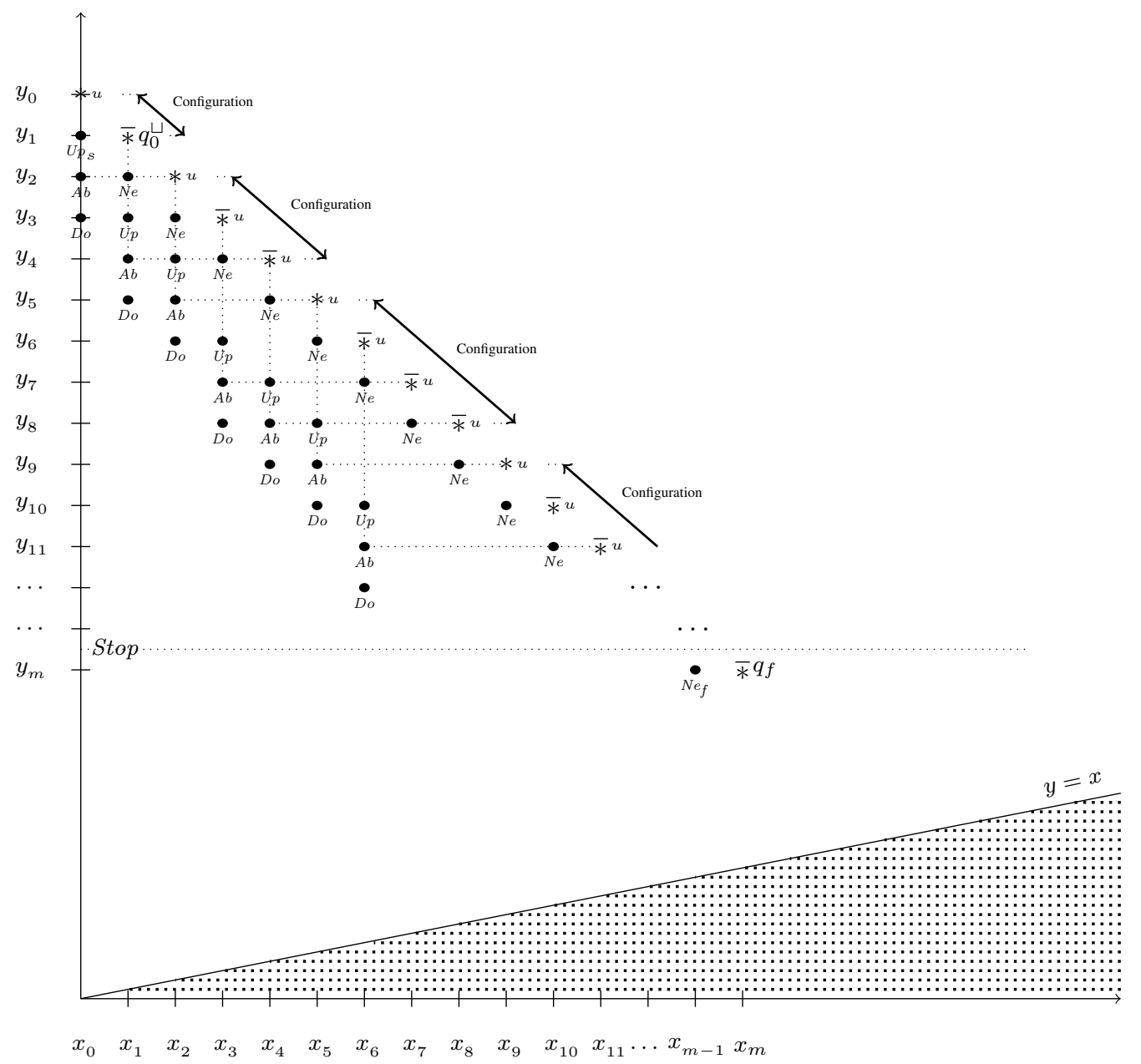

Figure 2. Configurations' structure.

state are set as follows:

$$
\begin{aligned}
& \phi_{2}=\text { Start } \wedge u \wedge * \wedge U(u \rightarrow(* \vee \bar{*})) \wedge\langle B\rangle \text { Stop } \wedge \\
& U(\text { Stop } \rightarrow([E] \text { Stop } \wedge[B][E] \text { Stop })) \\
& \phi_{3}=I_{d o}\left(\text { Start, } U p_{s}\right) \wedge I_{d o}(u, N e ?) \wedge I_{r i}(N e, u) \wedge \\
& I_{d o}(U p, A b ?) \wedge I_{d o}\left(U p_{s}, A b\right) \wedge I_{d o}(\text { Start }, N e) \\
& I_{d o}(A b, D o) \wedge I_{d o}\left(\text { Start }, N e_{s}\right) \wedge I_{r i}\left(N e_{s}, q_{0}^{\sqcup}\right) \wedge \\
& I_{r i}\left(N e_{f}, q_{f}\right) \\
& \phi_{4}=U((N e ? \wedge \neg S t o p) \rightarrow N e) \wedge \\
& U\left((N e ? \wedge \text { Stop }) \rightarrow N e_{f}\right) \wedge U\left(q_{f} \rightarrow u_{f}\right) \\
& U\left(\left(N e_{s} \wedge N e_{f}\right) \rightarrow \perp\right) \wedge U\left((u \wedge \neg \text { Stop }) \rightarrow \neg q_{f}\right) \\
& \phi_{5}=\bigwedge_{l \in \mathrm{L}} U(l \rightarrow \bar{*}) \wedge \bigwedge_{l \neq l^{\prime}} U\left(\left(l \wedge l^{\prime}\right) \rightarrow \perp\right) \\
& \phi_{6}=U((* \wedge \bar{*}) \rightarrow \perp)
\end{aligned}
$$

Lemma 2: Let us assume that $M,[x, y] \Vdash \phi_{1} \wedge \ldots \wedge \phi_{6}$. Then, there exist two finite sequences $x=x_{0}<x_{1}<\ldots<$ $x_{m}$ and $y=y_{0}>y_{1}>\ldots>y_{m}$ such that:

1) $M,\left[x_{0}, y_{0}\right] \Vdash u$ and if $M,\left[x_{i}, y_{i}\right] \Vdash u$, then either $M,\left[x_{i+1}, y_{i+1}\right] \Vdash u$ or $M,\left[x_{i+1}, y_{i+1}\right] \Vdash q_{f} \wedge u_{f}$ and $m=i+1$

2) for all $i<m$, either $M,\left[x_{i}, y_{i}\right] \Vdash *$ or $M,\left[x_{i}, y_{i}\right] \Vdash \bar{*}$.

$3)$ if $M,[x, y] \Vdash I_{r i}(p, q)$ and $M,\left[x_{i}, y_{j}\right] \Vdash p$, then $M,\left[x_{i+1}, y_{j}\right] \Vdash q ;$

4) if $M,[x, y] \Vdash I_{d o}(p, q)$ and $M,\left[x_{i}, y_{j}\right] \Vdash p$, then $M,\left[x_{i}, y_{j+1}\right] \Vdash q$.

Proof: The initial elements of the sequences are the two points $x=x_{0}$ and $y=y_{0}$ such that $M,\left[x_{0}, y_{0}\right] \Vdash$ $\phi_{1} \wedge \ldots \wedge \phi_{5}$. By $\phi_{2}$, it holds that $M,\left[x_{0}, y_{0}\right] \Vdash u$. Thanks to $\phi_{3}$, Lemma 1 , and Definition 2, if we put $y_{1}=$ $\operatorname{next}_{v}\left(y_{0}\right)$ and $x_{1}=\operatorname{next}_{h}\left(x_{0}\right)$, then $M,\left[x_{0}, y_{1}\right] \Vdash N e$ and $M,\left[x_{1}, y_{1}\right] \Vdash u$. Assume now that we have already generated the sequences $x_{0}<x_{1}<x_{2}<\ldots<x_{i}$ and $y_{0}>y_{1}>y_{2}>\ldots>y_{i}$ such that $M,\left[x_{i}, y_{i}\right] \Vdash u$. Again by $\phi_{3}$, following the same reasoning path as above, we define $y_{i+1}=\operatorname{next}_{v}\left(y_{i}\right)$ such that $M,\left[x_{i}, y_{i+1}\right] \Vdash N e$ ?. Now we have to consider two possibilities: if $M,\left[x_{i}, y_{i+1}\right] \Vdash \neg S t o p$, then we apply $\phi_{4}$ and $\phi_{3}$ to obtain $x_{i+1}=\operatorname{next}_{h}\left(x_{i}\right)$ such that $M,\left[x_{i+1}, y_{i+1}\right] \Vdash u$; otherwise, if $M,\left[x_{i}, y_{i+1}\right] \Vdash$ Stop, 
then we apply $\phi_{4}$ and $\phi_{3}$, and we put $x_{i+1}=\operatorname{next}_{h}\left(x_{i}\right)$ so that $M,\left[x_{i+1}, y_{i+1}\right] \Vdash q_{f}$. Moreover, we set $m=i+1$. The existence of such an $m>1$ is guaranteed by $\phi_{2}$ and $\phi_{5}$, by the construction of the sequences, and by the fact that we are dealing with the discrete/finite case, thus proving (1). Finally, (2) is a direct consequence of (1), the last clause of $\phi_{2}$, and $\phi_{6}$, while (3) and (4) easily follow from the definition of the sequence, Lemma 1, and Definition 2.

We require $A b$ and $A b_{f}$ to connect $*$-intervals with *intervals, and similarly for $\bar{*}$-intervals:

$$
\begin{aligned}
\phi_{7}= & U\left((u \wedge *) \rightarrow[B] S_{1}\right) \wedge U\left(\left(S_{1} \wedge A b\right) \rightarrow[E] S_{2}\right) \wedge \\
& U\left(\left(S_{2} \wedge u\right) \rightarrow *\right) \\
\phi_{8}= & U\left((u \wedge \bar{*}) \rightarrow[B] \overline{S_{1}}\right) \wedge U\left(\left(\overline{S_{2}} \wedge\left(u \vee u_{f}\right)\right) \rightarrow \bar{*}\right) \\
& U\left(\left(\overline{S_{1}} \wedge\left(A b \vee A b_{f}\right)\right) \rightarrow[E] \overline{S_{2}}\right)
\end{aligned}
$$

Now, we suitably place $A b, U p$, and $D o$ to create the chain of configurations, and $A b_{f}, U p_{f}$ to guarantee its finiteness:

$$
\begin{aligned}
\phi_{9}= & U\left((u \wedge *) \rightarrow[B] S_{3}\right) \wedge U\left((u \wedge \bar{*}) \rightarrow[B] S_{4}\right) \\
\phi_{10}= & U\left(\left(S_{3} \wedge D o\right) \rightarrow S_{5}\right) \wedge I_{r i}\left(S_{5}, U p ?\right) \\
\phi_{11}= & U\left(\left(S_{4} \wedge A b\right) \rightarrow S_{6}\right) \wedge I_{r i}\left(S_{6}, U p\right) \\
\phi_{12}= & U\left(\left((u \wedge *) \rightarrow u_{*}\right) \wedge I_{d o}\left(u_{*}, N e_{*}\right) \wedge I_{r i}\left(N e_{*}, \bar{*}\right)\right. \\
\phi_{13}= & U(((U p ? \wedge \neg S t o p) \rightarrow U p) \wedge \\
& U\left(\left((U p ? \wedge S t o p) \rightarrow U p_{f}\right) \wedge\right. \\
& U((A b ? \wedge \neg \text { Stop }) \rightarrow A b) \wedge \\
& U\left(\left((A b ? \wedge \text { Stop }) \rightarrow A b_{f}\right)\right.
\end{aligned}
$$

Lemma 3: Let $M,[x, y] \Vdash \phi_{1} \wedge \ldots \wedge \phi_{13}$ and let $x=$ $x_{0}<x_{1}<\ldots<x_{m}$ and $y=y_{0}>y_{1}>\ldots>y_{m}$ be the two finite sequences whose existence is guaranteed by Lemma 2. Then, for each $0 \leq i<m$ :

1) if $M,\left[x_{i}, y_{i}\right] \Vdash u \wedge *$ and $M,\left[x_{i}, y_{j}\right] \Vdash A b$ for some $j>i(j+2<m)$, then it holds that $M,\left[x_{j}, y_{j}\right] \Vdash *$, $M,\left[x_{i+1}, y_{j+1}\right] \Vdash U p$, and $M,\left[x_{i+1}, y_{j+2}\right] \Vdash A b$;

2) if $M,\left[x_{i}, y_{i}\right] \Vdash u \wedge \bar{*}$ and $M,\left[x_{i}, y_{j}\right] \Vdash A b$ for some $j>i(j<m)$, then $M,\left[x_{j}, y_{j}\right] \Vdash \bar{*}, M,\left[x_{i+1}, y_{j}\right] \Vdash$ $U p$, and $M,\left[x_{i+1}, y_{j+1}\right] \Vdash A b \vee A b_{f}$;

$3)$ if $M,\left[x_{i}, y_{i}\right] \Vdash u \wedge *$, then $M,\left[x_{i+1}, y_{i+1}\right] \Vdash \bar{*}$.

Proof: Assume that $M,\left[x_{i}, y_{i}\right] \Vdash u \wedge *$ and that, for some $j>i(j+2<m), M,\left[x_{i}, y_{j}\right] \Vdash A b$. By applying the first conjunct of $\phi_{7}$, we can conclude that $M,\left[x_{i}, y_{j}\right] \Vdash$ $S_{1} \wedge A b$. Then, by the second conjunct of $\phi_{7}$, it holds that $M,\left[x_{j}, y_{j}\right] \Vdash S_{2} \wedge u$, which implies (by the third conjunct of $\phi_{7}$ ) that $M,\left[x_{j}, y_{j}\right] \Vdash *$. Moreover, since $M,\left[x_{i}, y_{j}\right] \Vdash A b$ and, by $\phi_{3}, M,[x, y] \Vdash I_{d o}(A b, D o)$, we apply Lemma 2 (point (3)) to conclude that $M,\left[x_{i}, y_{j+1}\right] \Vdash D o$ and thus, by the first conjunct of $\phi_{9}, M,\left[x_{i}, y_{j+1}\right] \Vdash S_{3}$. Hence, we can apply the first conjunct of $\phi_{10}$ to prove that $M,\left[x_{i}, y_{j+1}\right] \Vdash$ $S_{5}$. By the second conjunct of $\phi_{10}, \phi_{13}$, and Lemma 2 (point (2)), we have that $M,\left[x_{i+1}, y_{j+1}\right] \Vdash U p$ and, by $\phi_{3}, \phi_{13}$, and Lemma 2 (point (3)), we have that $M,\left[x_{i+1}, y_{j+2}\right] \Vdash A b$, which completes the proof of (1). To prove (2), we reason as in (1) by using $\phi_{8}$ and $\phi_{11}$, instead of $\phi_{7}$ and $\phi_{10}$, . Finally, property (3) is a direct consequence of $\phi_{12}$ and Lemma 2 .

From the above lemmas, we obtain a structure (see Fig. 2), where, for any given unit $\left[x_{i}, y_{i}\right]$, we can define the correspondent unit as the unit $\left[x_{j}, y_{j}\right]$ such that $M,\left[x_{i}, y_{j}\right] \Vdash$ $A b \vee A b_{f}$. In this setting, we call a (non-final) configuration any interval $\left[x_{i}, y_{j}\right]$, with $j<m$, such that $U p \vee U p_{s}$ holds and the first unit $\left[x_{i}, y_{i}\right]$ satisfies $*$. The interval $\left[x_{s}, y_{m}\right]$, for some $s<m$, such that the first unit $\left[x_{s}, y_{s}\right]$ satisfies $*$ and $\left[x_{m}, y_{m}\right]$ satisfies $q_{f}$ is called final pseudo-configuration. Such an interval may not be a configuration, but it represents the initial part of the final configuration, as we will see later.

Lemma 4: Let $M,[x, y] \Vdash \phi_{1} \wedge \ldots \wedge \phi_{13}$ and let $x=$ $x_{0}<x_{1}<\ldots<x_{m}$ and $y=y_{0}>y_{1}>\ldots>y_{m}$ be the two finite sequences whose existence is guaranteed by Lemma 2. Then,

1) $\left[x_{0}, y_{1}\right]$ is the first configuration and there exists $0 \leq s<m$ such that $\left[x_{s}, y_{m}\right]$ is the final pseudoconfiguration;

2) for each $i, j \geq 0, j \leq m$, if $\left[x_{i}, y_{j}\right]$ is a configuration, then, for each $0<l \leq j-i$, the unit $\left[x_{i+l}, y_{i+l}\right]$ satisfies * (all units, but the first one, are $\bar{*}$ );

3) for each $i, j \geq 0, j<m$, if $\left[x_{i}, y_{j}\right]$ is a configuration, then the unit $\left[x_{i}, y_{i}\right]$ corresponds to the unit $\left[x_{j+1}, y_{j+1}\right]$;

4) for each $i, j \geq 0$ and $j<s-1$, if $\left[x_{i}, y_{j}\right]$ is a configuration, then, for each $0<l \leq j-i$, the unit $\left[x_{i+l}, y_{i+l}\right]$ corresponds to the unit $\left[x_{j+l+2}, y_{j+l+2}\right]$;

5) for each $i, j \geq 0$, if $\left[x_{i}, y_{j}\right]$ is a configuration and $j<$ $s-1$, then $\left[x_{j+1}, y_{2 j-i+2}\right]$ is a configuration (existence of the next configuration).

Proof: Let us prove point (1). First of all, $M,\left[x_{0}, y_{0}\right] \Vdash$ $* \wedge$ Start $\wedge I_{d o}\left(\right.$ Start, $\left.U p_{s}\right)$ implies $M,\left[x_{0}, y_{1}\right] \Vdash U p_{s}$, which proves that $\left[x_{0}, y_{1}\right]$ is the first configuration. Now, thanks to Lemma 2 (point (2)), we can define $s=\max \{i<$ $\left.m \mid M,\left[x_{i}, y_{i}\right] \Vdash *\right\}$, and thus, by Lemma 2 (point (1)), $M,\left[x_{m}, y_{m}\right] \Vdash q_{f}$, proving that $\left[x_{s}, y_{m}\right]$ is the final configuration. We prove points from (2) to (5) by induction on the configurations, the base case being $\left[x_{0}, y_{1}\right]$. Point (2) of the base case is a direct consequence of Lemma 3 (point (3). In order to prove (3) of the base case, we use $\phi_{2}, \phi_{3}$, and Lemma 2 (point (4)) to get $M\left[x_{0}, y_{2}\right] \Vdash A b$. To prove (4), consider the unit $\left[x_{1}, y_{1}\right]$. By point (3), we know that $M,\left[x_{0}, y_{2}\right] \Vdash A b$ and, by $\phi_{2}$, that $M,\left[x_{0}, y_{0}\right] \Vdash u \wedge *$. Now, if $m=3$, then $\left[x_{2}, y_{3}\right]$ is the last configuration and (4) is true. Otherwise, if $m \geq 4$, then, by Lemma 3 (point (1)), we have that $M,\left[x_{1}, y_{4}\right] \Vdash A b$ and thus $\left[x_{1}, y_{1}\right]$ corresponds to $\left[x_{4}, y_{4}\right]$. Finally, to prove point (5), consider the unit $\left[x_{2}, y_{2}\right]$. By point (3) and Lemma 3 (point (1)), we have that $M,\left[x_{2}, y_{2}\right] \Vdash *$. Moreover, since $M,\left[x_{1}, y_{1}\right] \Vdash \bar{*}$ and $M,\left[x_{1}, y_{4}\right] \Vdash A b$, by Lemma 3 (point (2)), we can conclude that $M,\left[x_{2}, y_{4}\right] \Vdash U p$, thus proving that $\left[x_{2}, y_{4}\right]$ 
is a configuration. As for the inductive case, assume that $\left[x_{i}, x_{j}\right]$ is a configuration and that (2)-(5) hold for the previous configuration $\left[x_{2 i-j}, y_{i-1}\right]$. By the definition of configuration, we have that $\left[x_{i}, y_{i}\right]$ is a $*$-interval, and thus, by Lemma 3 (point (3)), we have that $\left[x_{i+1}, y_{i+1}\right]$ is a $\bar{*}-$ interval, proving (2) when $l=1$. By the inductive hypothesis, $\left[x_{2 j-i+l}, y_{2 j-i+l}\right]$ is a $\bar{*}$-interval that corresponds to the unit $\left[x_{i+l+1}, y_{i+l+1}\right]$, for each $0<l \leq j-i-1$. By Lemma 3 (point (2)), $\left[x_{i+l+1}, y_{i+l+1}\right]$ must be a $\bar{*}$ interval, thus concluding the proof of (2). To prove (3), consider the last unit of the configuration $\left[x_{j}, y_{j}\right]$. By the inductive hypothesis, we have that it is in correspondence with the $\bar{*}$-unit $\left[x_{i-1}, y_{i-1}\right]$ of the previous configuration. This implies that $\left[x_{i-1}, y_{j}\right]$ is a $A b$-interval and thus, by Lemma 3 (point (2)), we have that $M,\left[x_{i}, y_{j+1}\right] \Vdash A b \vee A b_{f}$. By Lemma 3 (point (1)), we can conclude that $\left[x_{j+1}, y_{j+1}\right]$ is a $*$-unit in correspondence with $\left[x_{i}, y_{i}\right]$. We prove (4) by induction on $l$. Let $l=1$. By (3), we have that the $*-$ unit $\left[x_{i}, y_{i}\right]$ corresponds to $\left[x_{j+1}, y_{j+1}\right]$. This implies that $M,\left[x_{i}, y_{j+1}\right] \Vdash A b$ and thus, by Lemma 3 (point (1)), $\left[x_{i+1}, y_{j+3}\right] \Vdash A b$, proving that $\left[x_{i+1}, y_{i+1}\right]$ corresponds to $\left[x_{j+3}, x_{j+3}\right]$. Now, let $1<l \leq j-i$ and suppose that (4) holds for $l-1$. By the inductive hypothesis and (2), the $\bar{*}-$ unit $\left[x_{i+l-1}, y_{i+l-1}\right]$ corresponds to $\left[x_{j+l+1}, y_{j+l+1}\right]$. This implies that $M,\left[x_{i+l-1}, y_{j+l+1}\right] \Vdash A b \vee A b_{f}$ and thus, by Lemma 3 (point (2)), $\left[x_{i+l}, y_{j+l+2}\right] \Vdash A b \vee A b_{f}$, proving that $\left[x_{i+l}, y_{i+l}\right]$ corresponds to $\left[x_{j+l+2}, x_{j+l+2}\right]$. To prove (5), consider the unit $\left[x_{j+1}, y_{j+1}\right]$. By point (3), we have that $M,\left[x_{j+1}, y_{j+1}\right] \Vdash *$. Moreover, by point (2), it holds that $M,\left[x_{j}, y_{j}\right] \Vdash \bar{*}$ and, by (4), we get $M,\left[x_{j}, y_{2 j-i+2}\right] \Vdash A b$. Hence, by Lemma 3 (point (2)), we can conclude that $M,\left[x_{j+1}, y_{2 j-i+2}\right] \Vdash U p$, thus proving that $\left[x_{j+1}, y_{2 j-i+2}\right]$ is the next configuration.

The above lemma guarantees the existence of a finite sequence of configurations of the correct length. Notice that the final pseudo-configuration does not necessarily correspond to the final configuration of $\mathcal{M}_{T}$. Nevertheless, when the conjunction of all requirements is satisfiable, we can actually prove that it contains enough information to reconstruct the computation history of $\mathcal{M}_{T}$ including its final configuration.

Let $k_{0}, k_{1}, \ldots, k_{n}$ be the finite sequence of indices, which is inductively defined as follows: $k_{0}=0, k_{1}=2$, and, for each $i>1, k_{i}=2 k_{i-1}-k_{i-2}+1$. In such a way, for each $i>0,\left[x_{k_{i}}, y_{k_{i+1}-1}\right]$ turns out to be a configuration. In our construction, we cannot deal with neighboring units with a single application of a modal operator. Let us denote by $\mathrm{L}^{*}$ the set $\mathrm{L} \backslash\left\{q_{f}\right\}$ and let us use symbols of the form $\mathrm{L}^{t}=\left\{\left(l_{1}, l_{2}, l_{3}\right) \mid \forall i\left(1 \leq i \leq 3 \rightarrow l_{i} \in \mathrm{L}^{*}\right)\right\}$, considered as proposition letters, to overcome this technical problem by placing them over cells. We also introduce the auxiliary proposition letters $\left(l_{1}, l_{2}\right), \overline{\left(l_{1}, l_{2}\right)}, \overline{\overline{\left(l_{1}, l_{2}\right)}}, \overline{\left(l_{1}, l_{2}, l_{3}\right)}, R_{l}, A_{l}$, $A_{l, l^{\prime}}, N_{l, l^{\prime}}$, where $l_{i}, l, l^{\prime} \in \mathrm{L}^{*}$, and we impose the following conditions in order to create the triples:

$$
\begin{aligned}
& \phi_{14}=\bigwedge_{l \in \mathrm{L}^{*}} U\left(N e \wedge\langle E\rangle l \rightarrow R_{l}\right) \wedge \\
& \bigwedge_{l_{1}, l_{2} \in \mathrm{L}^{*}} U\left(\left(l_{1} \wedge\langle B\rangle R_{l_{2}}\right) \rightarrow\left(l_{1}, l_{2}\right)\right) \\
& \phi_{15}=\bigwedge_{l_{1}, l_{2} \in \mathrm{L}^{*}} U\left(\left(l_{1}, l_{2}\right) \rightarrow[B] \overline{\overline{\left(l_{1}, l_{2}\right)}} \wedge\right. \\
& \left.\left(\overline{\overline{\left(l_{1}, l_{2}\right)}} \wedge N e\right) \rightarrow \overline{\left(l_{1}, l_{2}\right)}\right) \\
& \phi_{16}=\bigwedge_{l_{1}, l_{2}, l_{3} \in \mathrm{L}^{*}}\left(U \left(\left(\overline{\left(l_{1}, l_{2}\right)} \wedge\langle E\rangle\left(l_{2}, l_{3}\right)\right) \rightarrow\right.\right. \\
& \left.[E] \overline{\left(l_{1}, l_{2}, l_{3}\right)}\right) \wedge \\
& \left.U\left(\left(\overline{\left(l_{1}, l_{2}, l_{3}\right)} \wedge u\right) \rightarrow\left(l_{1}, l_{2}, l_{3}\right)\right)\right) \\
& \phi_{17}=\bigwedge_{l \in L^{*}} U\left(\left(A_{l} \wedge A b\right) \rightarrow\langle E\rangle l\right) \\
& \phi_{18}=\bigwedge_{l, l^{\prime} \in \mathrm{L}^{*}} U\left(A_{l, l^{\prime}} \wedge A b \rightarrow[E] N_{l, l^{\prime}}\right) \\
& \phi_{19}=\bigwedge_{l, l^{\prime} \in \mathrm{L}^{*}} U\left(\left(u \wedge\langle B\rangle N_{l, l^{\prime}}\right) \rightarrow l\right) \\
& \phi_{20}=\bigwedge_{l, l^{\prime} \in \mathrm{L}^{*}} U\left(\left(N_{l, l^{\prime}} \wedge N e\right) \rightarrow\langle E\rangle l^{\prime}\right)
\end{aligned}
$$

To establish the proper link between $\mathcal{M}_{T}$-configurations and configurations, we make use of the following notions.

Definition 3: We say that an $\mathcal{M}_{T}$-configuration is initial (resp., final) if its state is $q_{0}$ (resp., $q_{f}$ ). For any two $\mathcal{M}_{T^{-}}$ configurations $C, C^{\prime}$, we say that $C^{\prime}$ is the $\mathcal{M}_{T}$-successor of $C$ if and only if $C^{\prime}$ is obtained by $C$ after exactly one application of $\delta$. Finally, a configuration is said to be coherent if and only if (i) there exists exactly one cell labeled by a proposition symbol representing the current state, (ii) every non-*-interval is a cell, and (iii) the content of every cell is unique.

The following formulae are used to ensure that the correct transitions take place:

$$
\begin{aligned}
\phi_{21}= & \bigwedge_{\left(c_{1}, c_{2}, c_{3}\right) \in \mathrm{L}_{t}, c_{3} \neq *} U\left(\left(c_{1}, c_{2}, c_{3}\right) \rightarrow[B] A_{c_{2}}\right) \\
\phi_{22}= & \bigwedge_{\left(*, c_{2}, c_{3}\right) \in \mathrm{L}_{t}} U\left(\left(*, c_{2}, c_{3}\right) \rightarrow[B] A_{\sqcup, c_{2}}\right) \\
\phi_{23}= & \bigwedge_{\substack{\delta(q, c)=\left(q^{\prime}, c^{\prime}, R\right) \\
\left(c_{1}, q^{c}, c_{3}\right) \in \mathrm{L}_{t}, c_{1} \neq *}} U\left(\left(c_{1}, q^{c}, c_{3}\right) \rightarrow[B] A_{c^{\prime}}\right) \\
\phi_{24}(q, c)=\left(q^{\prime}, c^{\prime}, R\right) & \bigwedge_{\left(*, q^{c}, c_{3}\right) \in \mathrm{L}_{t}, c_{3} \neq *} U\left(\left(*, q^{c}, c_{3}\right) \rightarrow[B] A_{\sqcup, c^{\prime}}\right) \\
\phi_{25}= & \bigwedge_{(q, c)=\left(q^{\prime}, c^{\prime}, R\right)} U\left(\left(c_{1}, q^{c}, *\right) \rightarrow[B] A_{q^{\prime c^{\prime}}}\right) \\
& \bigwedge_{\left(c_{1}, q^{c}, *\right) \in \mathrm{L}_{t}, c_{1} \neq *}
\end{aligned}
$$




$$
\begin{aligned}
& \phi_{26}=\bigwedge_{\substack{\left(*, q^{c}, *\right) \in \mathrm{L}_{t} \\
\delta(q, c)=\left(q^{\prime}, c^{\prime}, R\right)}}^{\delta(q, c)=\left(q^{\prime}, c^{\prime}, R\right)} U\left(\left(*, q^{c}, *\right) \rightarrow[B] A_{q^{\prime c^{\prime}}}\right) \\
& \phi_{27}=\bigwedge_{\left(q^{c}, c_{2}, c_{3}\right) \in \mathrm{L}_{t}, c_{2} \neq *} U\left(\left(q^{c}, c_{2}, c_{3}\right) \rightarrow[B] A_{q^{\prime c_{2}}}\right) \\
& \left.\phi_{28}=\bigwedge_{\left(c_{1}, c_{2}, q^{c}\right) \in \mathrm{L}_{t}, c_{1} \neq *}^{\delta(q, c)=\left(q^{\prime}, c^{\prime}, R\right)} U\left(\left(c_{1}, c_{2}, q^{c}\right) \rightarrow[B] A_{c_{2}}\right)\right) \\
& \phi_{29}=\bigwedge_{\left(*, c_{2}, q^{c}\right) \in \mathrm{L}_{t}}^{\delta(q, c)=\left(q^{\prime}, c^{\prime}, R\right)} U\left(\left(*, c_{2}, q^{c}\right) \rightarrow[B] A_{\sqcup, c_{2}}\right) \\
& \phi_{30}=\bigwedge_{\left(c_{1}, q^{c}, c_{3}\right) \in \mathrm{L}_{t}, c_{1} \neq *}^{\delta(q, c)=\left(q^{\prime}, c^{\prime}, L\right)} U\left(\left(c_{1}, q^{c}, c_{3}\right) \rightarrow[B] A_{c^{\prime}}\right) \\
& \phi_{31}=\bigwedge_{\left(*, q^{c}, c_{3}\right) \in \mathrm{L}_{t}}^{\delta(q, c)=\left(q^{\prime}, c^{\prime}, L\right)} U\left(\left(*, q^{c}, c_{3}\right) \rightarrow[B] A_{q^{\prime} \sqcup, c^{\prime}}\right) \\
& \phi_{32}=\bigwedge_{\left(q^{c}, c_{2}, c_{3}\right) \in \mathrm{L}_{t}}^{\delta(q, c)=\left(q^{\prime}, c^{\prime}, L\right)} U\left(\left(q^{c}, c_{2}, c_{3}\right) \rightarrow[B] A_{c_{2}}\right) \\
& \phi_{33}=\bigwedge_{\left(c_{1}, c_{2}, q^{c}\right) \in \mathrm{L}_{t}, c_{2} \neq *}^{\delta(q, c)=\left(q^{\prime}, c^{\prime}, L\right)} U\left(\left(c_{1}, c_{2}, q^{c}\right) \rightarrow[B] A_{q^{\prime c_{2}}}\right) \\
& \phi_{34}=\bigwedge_{\left(*, c_{2}, q^{c}\right) \in \mathrm{L}_{t}}^{\delta(q, c)=\left(q^{\prime}, c^{\prime}, L\right)} U\left(\left(*, c_{2}, q^{c}\right) \rightarrow[B] A_{\sqcup, q^{\prime} c_{2}}\right)
\end{aligned}
$$

Lemma 5: Let $M,[x, y] \Vdash \phi_{1} \wedge \ldots \wedge \phi_{34}$ and consider the finite sequence of indexes $k_{0}, k_{1}, \ldots k_{n}$ whose existence is guaranteed by Lemma 4 . Then,

1) the interval $\left[x_{k_{0}}, y_{k_{1}-1}\right]$ represents the initial $\mathcal{M}_{T^{-}}$ configuration of the Turing Machine $\mathcal{M}_{T}$ with empty input and it is coherent.

2) for each $0<i<n$, the interval $\left[x_{k_{i}}, y_{k_{i+1}-1}\right]$ is coherent and it is the $\mathcal{M}_{T}$-successor of (the $\mathcal{M}_{T}$-configuration represented by) the interval $\left[x_{k_{i-1}}\right.$, $\left.y_{k_{i}-1}\right]$.

Proof: As for (1), the fact that the interval $\left[x_{k_{0}}, y_{k_{1}-1}\right]=\left[x_{0}, y_{1}\right]$ represents the initial configuration of the Turing Machine is a direct consequence of Lemma 4 (point (1)). Its coherence is guaranteed by Lemma 4 (point (2)) and $\phi_{6}$.

To prove (2), let us consider an index $i>0$ and suppose that the property holds for the index $i-1$ (when $i=1$, we assume that (1) holds). Let $C$ be the $\mathcal{M}_{T}$-configuration represented by the interval $\left[x_{k_{i-1}}, y_{k_{i}-1}\right]$, which is coherent by the inductive hypothesis. Assume that the state in $C$ is $q$ and that the head is reading the $l$-th cell, labeled with $c \neq *$. There are several cases to take into consideration, depending on the movement required by $\delta$ and the position of the head in the tape. We recall here that we assumed the tape of the Turing Machine to be infinite to the left and finite to the right: at every transition, we add a new blank cell at the beginning of the configuration.

(a) $\delta(q, c)=\left(q^{\prime}, c^{\prime}, R\right)$ and neither the $(l-1)$-th nor the $(l+1)$-th unit is $*$. By $\phi_{14}, \phi_{15}$, and $\phi_{16}$, the $l$-th unit is labeled with $\left(c_{1}, q^{c}, c_{3}\right)$, for some $c_{1}, c_{3} \neq *$. By $\phi_{23}$ and $\phi_{17}$, the corresponding unit, that is, the $(l+1)$-th unit, on the configuration $\left[x_{k_{i}}, y_{k_{i+1}-1}\right]$ is labeled with $c^{\prime}$. By $\phi_{14}$, $\phi_{15}$, and $\phi_{16}$, it follows that the $(l-1)$-th unit is labeled with $\left(c_{0}, c_{1}, q^{c}\right)$, for some $c_{0}$. If $c_{0} \neq *$, then $\phi_{28}$ and $\phi_{17}$ apply, and thus the $l$-th unit of $\left[x_{k_{i}}, y_{k_{i+1}-1}\right]$ is labeled with $c_{1}$. On the other hand, if $c_{0}=*$, then $\phi_{29}, \phi_{18}, \phi_{19}$, and $\phi_{20}$ apply, implying that the second unit of $\left[x_{k_{i}}, y_{k_{i+1}-1}\right]$ is labeled with $c_{1}$ and that the (new) first unit of $\left[x_{k_{i}}, y_{k_{i+1}-1}\right]$ is labeled with $\sqcup$. Similarly, the $(l+1)$-th unit is labeled with $\left(q^{c}, c_{3}, c_{4}\right)$, for some $c_{4}$, and, by $\phi_{27}$ and $\phi_{17}$, we have that the $(l+2)$-th unit of $\left[x_{k_{i}}, y_{k_{i+1}-1}\right]$ is labeled with $q^{\prime c_{3}}$.

(b) $\delta(q, c)=\left(q^{\prime}, c^{\prime}, R\right)$, the $(l-1)$-th unit is $*$, and the $(l+1)$-th unit is not $*$. It immediately follows that $l=1$. By $\phi_{14}, \phi_{15}$, and $\phi_{16}$, the first unit of the $i$-1-th configuration is labeled with $\left(*, q^{c}, c_{3}\right)$, for some $c_{3} \neq *$. By $\phi_{24}, \phi_{18}, \phi_{19}$, and $\phi_{20}$, the second unit of the configuration $\left[x_{k_{i}}, y_{k_{i+1}-1}\right]$ is labeled with $c^{\prime}$, while the first unit is $\sqcup$. Similarly, the second unit of the $i-1$-th configuration is labeled with $\left(q^{c}, c_{3}, c_{4}\right)$, for some $c_{4}$, and, by $\phi_{27}$ and $\phi_{17}$, we have that the third unit of $\left[x_{k_{i}}, y_{k_{i+1}-1}\right]$ is labeled with $q^{\prime c_{3}}$.

(c) $\delta(q, c)=\left(q^{\prime}, c^{\prime}, R\right)$, the $(l-1)$-th unit is not $*$, and the $(l+1)$-th unit is $*$. In this case, we proceed as before, only applying $\phi_{25}$ to set the unit corresponding to the $l$-th one, and $\phi_{28}$ or $\phi_{29}$ to set the one corresponding to the $(l-1)$-th unit.

(d) $\delta(q, c)=\left(q^{\prime}, c^{\prime}, R\right)$ and both the $(l-1)$-th and the $(l+1)$-th unit are $*$. It immediately follows that $l=1$ and $i-1=0$ ( $C$ is the initial configuration). By $\phi_{14}$ and $\phi_{15}$, the first unit of the initial configuration is labeled with $\left(*, q^{c}, *\right)$, with $c=\sqcup$. By $\phi_{26}, \phi_{18}, \phi_{19}$, and $\phi_{20}$, the second unit of the configuration $\left[x_{k_{1}}, y_{k_{2}-1}\right]$ is labeled with $c^{\prime}$, while the first unit is $\sqcup$.

(e) $\delta(q, c)=\left(q^{\prime}, c^{\prime}, L\right)$ and the $(l-1)$-th unit is not $*$. By $\phi_{14}$ and $\phi_{15}$, the $l$-th unit is labeled with $\left(c_{1}, q^{c}, c_{3}\right)$, for some $c_{1}, c_{3}$ with $c_{1} \neq *$. By $\phi_{30}$ and $\phi_{17}$, the corresponding unit, that is, the $(l+1)$-th unit in the $i$-th configuration $\left[x_{k_{i}}, y_{k_{i+1}-1}\right]$, is labeled with $c^{\prime}$. Since the $(l-1)$-th unit is labeled with $\left(c_{0}, c_{1}, q^{c}\right)$, for some $c_{0}$, if $c_{0} \neq *$, then $\phi_{33}$ and $\phi_{17}$ apply, and thus the $l$-th unit of $\left[x_{k_{i}}, y_{k_{i+1}-1}\right]$ is labeled with $q^{\prime c_{1}}$. On the other hand, if $c_{0}=*$, then $\phi_{34}, \phi_{18}, \phi_{19}$, and $\phi_{20}$ apply, forcing the second unit of $\left[x_{k_{i}}, y_{k_{i+1}-1}\right]$ to be labeled with $q^{\prime c_{1}}$ and the (new) first one with $\sqcup$. Similarly, the $(l+1)$-th unit is labeled with $\left(q^{c}, c_{3}, c_{4}\right)$, for some $c_{4}$, and, by $\phi_{32}$ and $\phi_{17}$, we have that the $(l+2)$-th unit of $\left[x_{k_{i}}, y_{k_{i+1}-1}\right]$ is labeled with $c_{3}$.

(f) $\delta(q, c)=\left(q^{\prime}, c^{\prime}, L\right)$ and the $(l-1)$-th unit is $*$. We 
proceed as in case (e), but using $\phi_{32}$ and $\phi_{33}$.

Finally, by the coherence of $\left[x_{k_{i-1}}, y_{k_{i}-1}\right]$, which holds by the inductive hypothesis, every unit before the $(l-1)$-th one (excluding the 0 -th unit) is a $\bar{*}$ unit, as well as every unit after the $(l+1)$-th one. Therefore, $\phi_{14}$ and $\phi_{15}$ apply, and, by $\phi_{21}$ and $\phi_{22}$, their content is preserved, guaranteeing both the coherency of $\left[x_{k_{i}}, y_{k_{i+1}-1}\right]$ and the fact that it is the $\mathcal{M}_{T}$-successor of $\left[x_{k_{i-1}}, y_{k_{i}-1}\right]$.

Theorem 1: Let $\mathcal{M}_{T}$ be a deterministic Turing Machine. Then, $\mathcal{M}_{T}$ converges on empty input if and only if the reflexive $\mathrm{BE}_{\mathrm{HS}}$-formula

$$
\text { Halts }^{r e}=\phi_{1} \wedge \ldots \wedge \phi_{34}
$$

is satisfiable on a model built over a finite or a discrete linear order.

Proof: Let us assume that the $C$-formula Halts is satisfiable. Then, by Lemma 5, there exists a sequence of $\mathcal{M}_{T}$-configurations represented by the intervals $\left[x_{k_{0}}, y_{k_{1}-1}\right]$, ... $\left[x_{k_{n-1}}, y_{k_{n-1}}\right]$ which encodes the computation history of $\mathcal{M}_{T}$. Moreover, by Lemma 4 (point (1)), $\left[x_{s}, y_{m}\right]$ represents the final pseudo-configuration, where $s=k_{n}$. In order to construct the final $\mathcal{M}_{T}$-configuration, we have to consider the units corresponding to the units of the previous $\mathcal{M}_{T^{-}}$ configuration $\left[x_{k_{n-1}}, y_{k_{n}-1}\right]$ which are not in $\left[x_{s}, y_{m}\right]$. By hypothesis, it holds that, for some $k_{n-1}<r \leq k_{n}-1$, the unit $\left[x_{r}, y_{r}\right]$ satisfies $q_{c}$ and either $\delta(q, c)=\left(q_{f}, c^{\prime}, L\right)$ or $\delta(q, c)=\left(q_{f}, c^{\prime}, R\right)$, for some $c^{\prime} \in\{0,1\}$. Let us assume that $\delta(q, c)=\left(q_{f}, c^{\prime}, L\right)$ (the other case can be dealt with in a similar way). In this case, proceeding as in the proof of Lemma 4 (points (3) and (4)), we can show that $\left[x_{r-1}, y_{r-1}\right]$ corresponds to $\left[x_{m}, y_{m}\right] \mathrm{m}$ which satisfies $q_{f}$, and that every unit $\left[x_{j}, y_{j}\right]$, with $j \leq r-1$, has a corresponding unit in $\left[x_{s}, y_{m}\right]$. Now, we only need to use $\delta$ in order to extend the final pseudo-configuration $\left[x_{s}, y_{m}\right]$ to the final $\mathcal{M}_{T}$-configuration $\left[x_{s}^{\prime}, y_{p}^{\prime}\right]$, with $p \geq m$, in such a way that it contains all the corresponding elements from the previous configuration, in particular those corresponding to the units $\left[x_{j}, y_{j}\right]$, for $j \geq r$, that were not included in $\left[x_{s}, y_{m}\right]$, thus proving that the satisfiability of the $C$-formula Halts implies that $\mathrm{A}$ converges. The opposite implication is straightforward.

Corollary 1: The satisfiability problem for reflexive $\mathrm{BE}_{\mathrm{HS}}$ and for $\mathrm{C}$, interpreted on finite or discrete linear orders, is undecidable.

\section{Generalization to the Infinite CASE}

When we deal with linear orders which are infinite, but not necessarily discrete, we have to consider the non-halting problem for a Turing Machine and to modify the formulae $\phi_{2}, \ldots, \phi_{13}$ in order to obtain an infinite sequence of units:

$$
\begin{aligned}
\psi_{1}= & \text { Start } \wedge u \wedge * \wedge I_{d o}(\text { Start }, U p) \wedge \\
& I_{d o}\left(\text { Start }, N e_{s}\right) \wedge I_{r i}\left(N e_{s}, q_{0}^{\sqcup}\right) \\
\psi_{2}= & I_{d o}(u, N e) \wedge I_{r i}(N e, u) \wedge I_{d o}(U p, A b) \wedge \\
& I_{d o}(A b, D o) \\
\psi_{3}= & \bigwedge_{l \in \mathrm{L}} U(l \rightarrow \bar{*}) \wedge \wedge_{l \neq l^{\prime}} U\left(\left(l \wedge l^{\prime}\right) \rightarrow \perp\right) \\
\psi_{4}= & U\left((u \wedge *) \rightarrow[B] S_{1}\right) \wedge \\
& U\left(\left(S_{1} \wedge A b\right) \rightarrow[E] S_{2}\right) \wedge U\left(\left(S_{2} \wedge u\right) \rightarrow *\right) \\
\psi_{5}= & U\left((u \wedge \bar{A}) \rightarrow[B] \overline{S_{1}}\right) \wedge \\
& U\left(\left(\overline{S_{1}} \wedge A b\right) \rightarrow[E] \overline{S_{2}}\right) \wedge U\left(\left(\overline{S_{2}} \wedge u\right) \rightarrow \bar{*}\right) \\
\psi_{6}= & U((* \wedge \bar{*}) \rightarrow \perp) \\
\psi_{7}= & U\left((u \wedge *) \rightarrow[B] S_{3}\right) \wedge U\left((u \wedge \bar{*}) \rightarrow[B] S_{4}\right) \\
\psi_{8}= & U\left(\left(S_{3} \wedge D o\right) \rightarrow S_{5}\right) \wedge I_{r i}\left(S_{5}, U p\right) \\
\psi_{9}= & U\left(\left(S_{4} \wedge A b\right) \rightarrow S_{6}\right) \wedge I_{r i}\left(S_{6}, U p\right) \\
\psi_{10}= & U\left((u \wedge *) \rightarrow u_{*}\right) \wedge I_{d o}\left(u_{*}, N e_{*}\right) \wedge I_{r i}\left(N e_{*}, \bar{*}\right)
\end{aligned}
$$

Now, we can suitably adapt the statement of the theorem and prove it in a similar (but not identical) way. In particular, we cannot refer to the successor of a set anymore; instead, we have to build the $u$-chain step-by-step, by choosing the representative of a set at a certain stage and, then, possibly modifying that choice in the next stage, as shown by the following result which replaces Lemma 2 and Lemma 3.

Lemma 6: Let $M,[x, y] \Vdash \phi_{1} \wedge \psi_{1} \wedge \ldots \wedge \psi_{10}$. Then, there exist two infinite sequences $x=x_{0}<x_{1}<\ldots$ and $y=y_{0}>y_{1}>\ldots$ such that, for every $i \geq 0$,

1) $M,\left[x_{i}, y_{i}\right] \Vdash u$;

2) if $M,\left[x_{i}, y_{i}\right] \Vdash u \wedge *$, then $M,\left[x_{i+1}, y_{i+1}\right] \Vdash \bar{*}$;

3) there exists $j>i+1$ such that $M,\left[x_{i}, y_{j-1}\right] \Vdash U p$, $M,\left[x_{i}, y_{j}\right] \Vdash A b, M,\left[x_{i}, y_{j+1}\right] \Vdash D_{o}$, and:

a) if $M,\left[x_{i}, y_{i}\right] \Vdash *$, then $M,\left[x_{j}, y_{j}\right] \Vdash *$ and $M,\left[x_{i+1}, y_{j+1}\right] \Vdash U p$;

b) if $M,\left[x_{i}, y_{i}\right] \Vdash \bar{*}$, then $M,\left[x_{j}, y_{j}\right] \Vdash \bar{*}$ and $M,\left[x_{i+1}, y_{j}\right] \Vdash U p$.

Proof: By $\psi_{1}, \psi_{2}$, and $\psi_{10}$, it holds that $M,\left[x_{0}, y_{0}\right] \Vdash$ $u \wedge I_{d o}(u, N e) \wedge u_{*} \wedge I_{d o}\left(u_{*}, N e_{*}\right)$. Next, we apply twice Lemma 1 (for $I_{d o}$ ) to obtain $t, t^{\prime}<\operatorname{set}_{v}\left(y_{0}\right)$ such that $M,\left[x_{0}, t\right] \Vdash N e$ and $M,\left[x_{0}, t^{\prime}\right] \Vdash N e_{*}$. Now, let $y_{1}=$ $\max \left\{t, t^{\prime}\right\}$. By Lemma 1 (point (4)), we get $M,\left[x_{0}, y_{1}\right] \Vdash$ $N e \wedge N e_{*}$. Since $M,\left[x_{0}, y_{1}\right] \Vdash I_{r i}(N e, u) \wedge I_{r i}\left(N e_{*}, \bar{*}\right)$, we obtain $s, s^{\prime}>\operatorname{set}_{h}\left(x_{0}\right)$ such that $M,\left[s, y_{1}\right] \Vdash u$ and $M,\left[s^{\prime}, y_{1}^{\prime}\right] \Vdash \bar{*}$. We define now $x_{1}=\min \left\{s, s^{\prime}\right\}$ to get $M,\left[x_{1}, y_{1}\right] \Vdash u \wedge \bar{*}$.

We can inductively repeat the above reasoning step (or an easier one, in case $\left.M,\left[x_{i}^{\prime}, y_{i}^{\prime}\right] \Vdash u \wedge ₹\right)$ in order to obtain two infinite sequences $x=x_{0}<x_{1}<x_{2}<\ldots$ and $y=y_{0}>y_{1}>y_{2}>\ldots$ such that, for every $i \geq 0$, we have that $M,\left[x_{i}, y_{i}\right] \Vdash u$ and if $M,\left[x_{i}, y_{i}\right] \Vdash u \wedge *$, then $M,\left[x_{i+1}, y_{i+1}\right] \Vdash \bar{*}$, proving (1) and (2).

The proof of (3) is by induction on $i$. As for the base case, by $\psi_{1}$, we have that $M,\left[x_{0}, y_{0}\right] \Vdash$ Start $\wedge I_{d o}($ Start, $U p)$. Hence, by Lemma 1, there exists $t>\operatorname{set}_{v}\left(y_{0}\right)$ such that 
$M,\left[x_{0}, t\right] \Vdash U p$. We now re-define $y_{1}$ as the maximum between the previous value of $y_{1}$ and $t$, and apply again the same reasoning as in points (1) and (2) to re-construct the $u$-chain for every $i \geq 1^{2}$. The same reasoning process can be followed to determine $y_{2}, y_{3}$ such that $M,\left[x_{0}, y_{2}\right] \Vdash A b$ and $M,\left[x_{0}, y_{3}\right] \Vdash D o$, reconstructing again the $u$-chain for $i \geq 2$ and $i \geq 3$, respectively. Now, as $M,\left[x_{2}, y_{2}\right] \Vdash u$, we apply $\psi_{4}$ to get $M,\left[x_{2}, y_{2}\right] \Vdash *$. We can apply now $\psi_{7}$ and $\psi_{8}$ and reasoning as above we obtain $x_{1}$ such that $M,\left[x_{1}, y_{3}\right] \Vdash U p$, proving (3) in the base case. As for the inductive step, let us assume that (3) holds up to $i-1$ and let us prove it for $i$. By (1), we have that $M,\left[x_{i-1}, y_{i-1}\right] \Vdash u$ and, by the inductive hypothesis, there exists $j>i-1$ such that $M,\left[x_{i-1}, y_{j-1}\right] \Vdash U p, M,\left[x_{i-1}, y_{j}\right] \Vdash A b$, and $M,\left[x_{i-1}, y_{j+1}\right] \Vdash D o$. Moreover, if $M,\left[x_{i-1}, y_{i-1}\right] \Vdash \bar{*}$ (the other case is similar), then $M,\left[x_{j}, y_{j}\right] \Vdash \approx$ and $M,\left[x_{i}, y_{j}\right] \Vdash U p$. Now, we apply again $\psi_{2}$ and we proceed as in the base case to obtain $y_{j+1}, y_{j+2}$ (re-constructing the $u$-chain for all values greater than or equal to $j-1$ and $j-2$, respectively) such that $M,\left[x_{i}, y_{j+1}\right] \Vdash A b$ and $M,\left[x_{i}, y_{j+2}\right] \Vdash D o$. If $M,\left[x_{i}, y_{i}\right] \Vdash{ }^{*}$ (the other case is similar), then, by $\psi_{5}$, we get $M,\left[x_{j+1}, y_{j+1}\right] \Vdash \bar{*}$ and, by $\psi_{7}$ and $\psi_{9}$, we can determine $x_{i+1}$ such that $M,\left[x_{i+1}, y_{j+1}\right] \Vdash U p$, proving (3).

Minor changes to Lemmas 4 and 5 lead us to the final result.

Theorem 2: Let $\mathcal{M}_{T}$ be a deterministic Turing Machine. Then, $\mathcal{M}_{T}$ diverges on empty input if and only if the reflexive $\mathrm{BE}_{\mathrm{HS}}$-formula

NonHalts $=\phi_{1} \wedge \psi_{1} \wedge \ldots \wedge \psi_{10} \wedge \phi_{14} \wedge \cdots \wedge \phi_{34} \wedge U\left(q_{f} \rightarrow \perp\right)$

is satisfiable on a model built over the class of all linear orders or the class of all dense linear orders.

Corollary 2: The satisfiability problem for reflexive $\mathrm{BE}_{\mathrm{HS}}$ and for $\mathrm{C}$, interpreted in the class of all linear orders or the class of all dense linear orders, is undecidable.

\section{CONCLUSions}

In this paper, we traced Lodaya's steps [14] by analyzing the relationship between the fragments $D_{H S}, B E_{H S}$, and $C$ in the special case in which $\pi$ is not included in the language and $D_{H S}$ and $B E_{H S}$ are interpreted in a reflexive fashion. In their standard version (irreflexive fragments of HS and $C$ enriched with $\pi$ ), undecidability of $\mathrm{BE}_{\mathrm{HS}}$ in the finite/discrete case is a consequence of the undecidability of the $D_{H S}[15]$; in contrast, in the reflexive case, the undecidability area includes $\mathrm{BE}_{\mathrm{HS}}$, in any meaningful class of linear orders, but not $\mathrm{D}_{\mathrm{HS}}$, which is decidable at least in the finite/discrete case [16]. Our results close a major gap in the study, started in [12], of the fragments of Venema's CDT

\footnotetext{
${ }^{2}$ Thanks to Definition 1 and Lemma 1 (point (5)), this reconstruction is well defined as all the elements remain in the same set.
}

(which is reflexive in nature) devoid of $\pi$, as well as sharpen both the undecidability of PITL without locality principle and that of fragments of HS in their reflexive version.

It should be mentioned that there might be the possibility of designing a simpler undecidibility proof for $C$, devoid of $\pi$, based on a reduction from the undecidable satisfiability problem for irreflexive $D_{H S}$ over finite/discrete linear orders [15], which still exploits the ideas underlying our proof (in particular, Definition 1$)^{3}$. In any case, such a reduction would not work for reflexive $\mathrm{BE}_{\mathrm{HS}}$.

\section{ACKNOWLEDGMENTS}

The authors acknowledge the support from the Spanish Project TIN12-39353-C04-01 (G. Sciavicco and E. MuñozVelasco), the Spanish fellowship program 'Ramón y Cajal' RYC-2011-07821 (G. Sciavicco), and the GNCS project Algorithms for model checking and synthesis of safetycritical systems (A. Montanari).

\section{REFERENCES}

[1] J. F. Allen, "Maintaining knowledge about temporal intervals," Communications of the ACM, vol. 26, no. 11, pp. 832843, 1983.

[2] A. Artale, D. Bresolin, A. Montanari, V. Ryzhikov, and G. Sciavicco, "DL-lite and interval temporal logics: A marriage proposal," in Proc. of the 21st European Conference of Artificial Intelligence (ECAI), 2014, pp. 957-958.

[3] A. Artale and E. Franconi, "A temporal description logic for reasoning about actions and plans," Journal of Artificial Intelligence Reasoning, vol. 9, pp. 463-506, 1998.

[4] A. Artale, V. Ryzhikov, R. Kontchakov, and M. Zakharyaschev, "A cookbook for temporal conceptual data modelling with description logics," ACM Transaction on Computational Logic, vol. 15, no. 3, pp. 25:1-25:50, 2014.

[5] C. Bettini, "Time-dependent concepts: Representation and reasoning using temporal description logics," Data Knowedge Engeneering, vol. 22, no. 1, pp. 1-38, 1997.

[6] D. Bresolin, D. Della Monica, A. Montanari, P. Sala, and G. Sciavicco, "On the complexity of fragments of the modal logic of Allen's relations over dense structures," in Proc. of the 9th International Conference on Language and Automata Theory and Applications (LATA), ser. LNCS, no. 8977. Springer, 2015, pp. $511-523$.

[7] D. Bresolin, D. Della Monica, A. Montanari, and G. Sciavicco, "The light side of interval temporal logic: the BernaysSchönfinkel fragment of CDT," Annals of Mathematics and Artificial Intelligence, vol. 71, no. 1-3, pp. 11-39, 2014.

[8] D. Bresolin, D. D. Monica, A. Montanari, P. Sala, and G. Sciavicco, "Interval temporal logics over strongly discrete linear orders: Expressiveness and complexity," Theoretical Computer Science, vol. 560, pp. 269-291, 2014.

\footnotetext{
${ }^{3} \mathrm{We}$ would like to thank one of the anonymous reviewers for this suggestion.
} 
[9] D. Bresolin, A. Montanari, P. Sala, and G. Sciavicco, "What's decidable about Halpern and Shoham's interval logic? the maximal fragment ABBL," in Proc. of the 26th IEEE Symposium on Logic in Computer Science (LICS). IEEE Computer Society, 2011, pp. 387-396.

[10] A. Cau, H. Janicke, and B. C. Moszkowski, "Verification and enforcement of access control policies," Formal Methods in System Design, vol. 43, no. 3, pp. 450-492, 2013.

[11] J. Halpern and Y. Shoham, "A propositional modal logic of time intervals," Journal of the ACM, vol. 38, no. 4, pp. 935962, 1991

[12] I. Hodkinson, A. Montanari, and G. Sciavicco, "Non-finite axiomatizability and undecidability of interval temporal logics with C, D, and T," in Proc. of the17th EACSL Annual Conference on Computer Science Logic (CSL), ser. LNCS, vol. 5213. Springer, 2008, pp. 308-322.

[13] B. Jónsson, "Varieties of relation algebras," Algebra Universalis, vol. 15, no. 1, pp. 273-298, 1982.

[14] K. Lodaya, "Sharpening the undecidability of interval temporal logic," in Proc. of the 6th Asian Computing Science Conference, ser. LNCS, vol. 1961. Springer, 2000, pp. 290298.

[15] J. Marcinkowski and J. Michaliszyn, "The undecidability of the logic of subintervals," Fundamenta Informaticae, vol. 131, no. 2, pp. 217-240, 2014.

[16] A. Montanari, I. Pratt-Hartmann, and P. Sala, "Decidability of the logics of the reflexive sub-interval and super-interval relations over finite linear orders," in Proc. of the 17th International Symposium on Temporal Representation and Reasoning (TIME). IEEE Comp. Soc., 2010, pp. 27-34.

[17] A. Montanari, G. Puppis, and P. Sala, "A decidable spatial logic with cone-shaped cardinal directions," in Proc. of the18th EACSL Annual Conference on Computer Science Logic (CSL), ser. LNCS, vol. 5771. Springer, 2009, pp. 394-408.

[18] —, "Maximal decidable fragments of Halpern and Shoham's modal logic of intervals," in Proc. of the 37th International Colloquium on Automata, Languages and Programming - Part II (ICALP), ser. LNCS, vol. 6199. Springer, 2010, pp. $345-356$

[19] —- "Decidability of the interval temporal logic $A \bar{A} B \bar{B}$ over the rationals," in Proc. of the 39th International Symposium on Mathematical Foundations of Computer Science - Part I (MFCS), ser. LNCS, vol. 8634. Springer, 2014, pp. 451-463.

[20] B. C. Moszkowski, "Reasoning about digital circuits," Ph.D. dissertation, Dept. of Computer Science, Stanford University, Stanford, CA, 1983.

[21] _ - "Interconnections between classes of sequentially compositional temporal formulas," Information Processing Letters, vol. 113, no. 9, pp. 350-353, 2013.

[22] — "Compositional reasoning using intervals and time reversal," Annals of Mathematics and Artificial Intelligence, vol. 71, no. 1-3, pp. 175-250, 2014.
[23] I. Pratt-Hartmann, "Temporal prepositions and their logic," Artificial Intelligence, vol. 166, no. 1-2, pp. 1-36, 2005.

[24] A. Schmiedel, "Temporal terminological logic," in Proc. of the 8th National Conference on Artificial Intelligence (AAAI). AAAI Press, 1990, pp. 640-645.

[25] Y. Venema, "Expressiveness and completeness of an interval tense logic," Notre Dame Journal of Formal Logic, vol. 31, no. 4, pp. 529-547, 1990.

[26] — "A modal logic for chopping intervals," Journal of Logic and Computation, vol. 1, no. 4, pp. 453-476, 1991.

[27] C. Zhou and M. R. Hansen, Duration Calculus: A Formal Approach to Real-Time Systems, ser. EATCS: Monographs in Theoretical Computer Science. Springer, 2004. 